\title{
A NOVEL ANALYTICAL CONTROL OF A SINGLE ROTARY INVERTED PENDULUM UNDER INITIAL ANGULAR DISPERSIONS
}

\author{
Fawaz F. Al-Bakri', Salwan Obaid Waheed Khafaji ${ }^{*}$, Hasan H. Ali ${ }^{3}$ \\ ${ }^{1}$ Technical Institute of Babylon; Al-Furat Al-Awsat Technical University (ATU), Iraq. \\ ${ }^{2}$ University of Babylon, College of Engineering, Mechanical Department, Babylon, Iraq. \\ ${ }^{3}$ Directorate of Reconstruction and Projects; Ministry of Higher Education and Scientific Research, Baghdad, Iraq \\ *corresponding author: Salwan Obaid Waheed Khafaji \\ Email:sw9p4@mail.missouri.edu
}

\begin{abstract}
A nonlinear single rotary inverted pendulum is considered in this paper. A novel online algorithm, that is used to stabilize the rotary pendulum targeting the desired profiles based on analytical solutions, is presented. The new algorithm employs a single analytical function to create the pendulum angle and the velocity profiles. The reference pendulum angle is described by a series of time dependent exponential functions. Boundary conditions at the rest and the steady-state upright conditions are automatically satisfied. Moreover, target pendulum angle at the steady-state upright condition is ensured by evaluating one algorithm parameter (the quarter-way pendulum angle) for the reference pendulum angle and velocity functions. The quarter-way pendulum angle is numerically computed by integrating a simple link angular displacement differential equation. This new algorithm is capable of generating the well-suited torque that guarantees the stability of the system without utilizing any linear or nonlinear control methods. Furthermore, unlike the existing pendulum algorithms, the analytical scheme proposed in this work is able to successfully handle very large dispersions in initial pendulum angle while satisfying the desired states. Numerical simulations with random off-nominal conditions (with change in initial pendulum angle) are presented. The results demonstrated that the proposed algorithm is effective in performing the system stabilization with great performance characteristics.
\end{abstract}

Keywords: Nonlinear analysis, Inverted pendulum, Analytical control, Monte-Carlo simulation.

\section{Introduction}

Rotary pendulum systems have been of interest to many researchers in the recent decades due to their nonlinear and unstable nature and their wide range of applications including robotics and aerospace. Various controllers have been used such as PID, LQR, FSF, sliding mode, etc. The objective of those controllers was to stabilize the pendulum in its unstable upright position. The performance and robustness of the system under the existence of disturbances and parameter uncertainties were studied to assess the effectiveness of those controllers.

A mathematical model for an inverted pendulum system was presented in [1]. The pendulum links number was arbitrary. The second kind Lagrange equations were used along with Rayleigh dissipation function in the modeling process. Energy balance for the base and arbitrary links were implemented using MATLAB in form of symbolic MATLAB functions and a MATLAB GUI application. The responses of the open-loop system were used to validate the generated equations of motion.
In [2] a review of the single rotary inverted pendulum system is presented. The modeling and analysis of the pendulum system were included in the review in addition to the controller design that successfully stabilizes the pendulum system in the up-right unstable position. Various controller designs for inverted rotary pendulum system are presented in [3]. Proportional-integral-derivative (PID), two degrees of freedom (2DOF) PID, full state feedback (FSF), and linear quadratic regulator (LQR) controllers were considered for the swing up and upright modes. The results showed that the 2DOF PID controller provided a satisfactory performance. However, the system with 2DOF PID controller was not robust to parameter changes. The FST and LQR based controllers satisfy both the performance requirements and the robustness conditions.

Lyapunov theorem for stability was used in $[4,5]$ to stabilize a rotary pendulum system. Lyapunov function was assumed to be a logarithmic function which was shown to provide a better performance than the typical quadratic function. The results of the proposed controller were validated experimentally and compared with the LQR controller. 
In addition, the robustness of the system was assessed. A control design for a rotary pendulum was presented in [6]. PD cascade scheme was used for the pendulum swing up control while a fuzzy PD control was used for the system stabilization. The system was analyzed using Matlab. A switching criteria was introduced to switch between the two modes.

Full state controller was designed in [7] to stabilize a rotary pendulum in its upright mode. For controllers were considered based on different damping ratios. The system was analyzed using MATLAB and the results were validated experimentally. In [8], nonlinear and linearized models for a rotary inverted pendulum were studied. PID, FSF, and LQR controllers were considered. A comparison of the results was performed to assess how those controllers are accurate, efficient, and reliable. The results showed that the LQR has faster response, more robustness, and higher accuracy. An inverted pendulum control system was designed in [9]. The control objective was to stabilize the pendulum in the upright position. That objective was achieved by designing three controllers; one to destabilize the pendulum from its downward position and move it back and forth until it becomes close enough to the upright position, the second one is to stabilize it in the upright position, and the third one was to switch between the two controllers. Simulation was used to analyze the results and experiments were used to validate them.

Another kind of controllers that was implemented for a rotary pendulum is the adaptive neural based fuzzy inference system (ANFIS) presented in [10]. It is a combination of LQR and fuzzy controllers. The robustness of the system to change in the pendulum mass was studied and compared to that of the system with LQR controller which shows a performance improvement. Sliding mode variable structure control for a rotary inverted pendulum was presented in [11]. Two sliding surfaces were design depending on the system performance and a Lyapunov function was defined to control the system which provided satisfactory results. H-infinity controller for a rotary pendulum system was designed in [12]. Taylor series was used for the linearization purposes. Lyapunov analysis was used to prove the stability of the system. The results showed that the controller meets the stability and performance specifications.

This work proposes a novel online algorithm to stabilize the single rotary inverted pendulum analytically. The new algorithm uses a single analytical function to generate the pendulum angle and angular velocity profiles. The reference pendulum is parametrized by a six-term exponential function with time as the independent variable.

The tracking reference profiles are obtained by adopting a single free variable, the pendulum angle at the quarter-way time while achieving all the boundary conditions. Since the proposed exponential function provides a congruent reference pendulum angle with the actual profile, the reference torque can guarantee stability of the system with no control effort. Monte Carlo simulations demonstrated that the proposed algorithm successfully performs the stability of the system despite large dispersions in the initial pendulum angle.

\section{System Model}

The dynamics of a single rotary inverted pendulum are derived using Newton's laws of motion. The governing differential equations of motion are found in [13] and can be expressed as follows,

$$
\begin{aligned}
& \ddot{\theta}_{2}\left(m_{2} l_{2}^{2}+j_{1}\right)=-g m_{2} l_{2} \sin \theta_{2}-b_{2} \dot{\theta}_{2}-m_{2} l_{1} l_{2} \ddot{\theta}_{1} \cos \theta_{2} \\
& \ddot{\theta}_{1}\left(m_{2} l_{1}^{2}+j_{1}\right)=\tau-m_{2} l_{1} l_{2}\left(\ddot{\theta}_{2} \cos \theta_{2}-\dot{\theta}_{2}^{2} \sin \theta_{2}\right)-b_{1} \dot{\theta}_{1}
\end{aligned}
$$

where $\ddot{\theta}_{1}, \dot{\theta}_{1}, \theta_{1}$ are the link angular acceleration, velocity, and displacement, respectively, $\ddot{\theta}_{2}, \dot{\theta}_{2}, \theta_{2}$ are the pendulum angular acceleration, velocity, and displacement, respectively, $m_{2}(\mathrm{~kg})$ is the pendulum mass, $l_{1}$ and $l_{2}(\mathrm{~m})$ are the link and pendulum lengths, respectively, $j_{1}$ and $j_{2}\left(\mathrm{~kg} . \mathrm{m}^{2}\right)$ are the link and pendulum moment of inertias, respectively, $b_{1}$ and $b_{2}$ (N.m.s) are the frictions of the motor and the pendulum, respectively, $g\left(\mathrm{~m} / \mathrm{s}^{2}\right)$ is the gravitational acceleration, and eventually, $\tau$ (N.m) is the motor torque. Figure 1 shows the threedimensional schematic of the single rotary inverted pendulum. The single rotary inverted pendulum model is described by two second order differential equations. Hence, the system requires four state variables. The link angle, $\theta_{1}$, the link angular velocity, $\dot{\theta}_{1}$, the pendulum angle, $\theta_{2}$, and the pendulum angular velocity, $\dot{\theta}_{2}$ were selected as the four state variables.

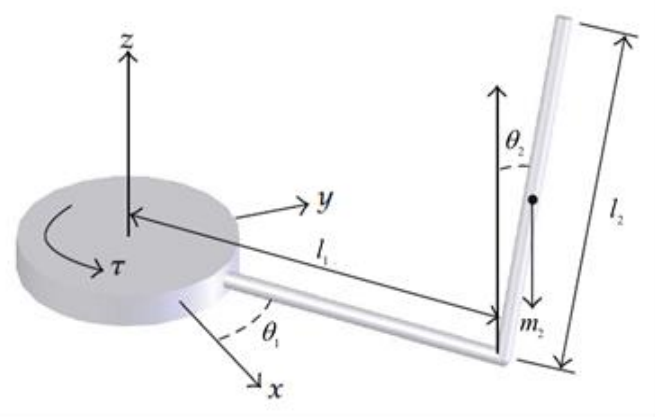

Figure 1: Schematic of single rotary inverted pendulum [14]

The applied motor torque $\tau$ is the input to the rotational inverted system. Suppose that 


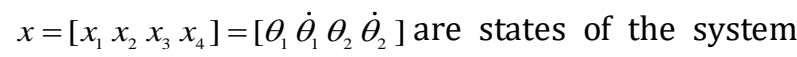
and $u=\tau$ is the system input. Next, the complete state space representation (SSR) of the single rotary inverted pendulum system can be obtained as follows,

$$
\begin{aligned}
& \dot{x}_{1}=x_{2} \\
& \dot{x}_{2}=\frac{\left(\begin{array}{l}
m_{2}^{2} l_{1} l_{2}^{2} g \sin x_{3} \cos x_{3}+m_{2} l_{1} l_{2} b_{2} x_{4} \cos x_{3}+ \\
m_{2} l_{1} l_{2}\left(m_{2} l_{2}^{2}+j_{2}\right) \sin x_{3}-b_{1}\left(m_{2} l_{2}^{2}+j_{2}\right) x_{2}+ \\
\left(m_{2} l_{2}^{2}+j_{2}\right) u
\end{array}\right)}{m_{2}^{2} l_{1}^{2} l_{2}^{2}+m_{2} j_{2} l_{1}^{2}+m_{2} l_{2}^{2} j_{1}+j_{1} j_{2}-m_{2}^{2} l_{1}^{2} l_{2}^{2} \cos ^{2} x_{3}} \\
& \dot{x}_{3}=x_{4} \\
& \dot{x}_{4}=-\left(\frac{\left(m_{2} l_{2} g \sin x_{3} b_{2} x_{4}\right)}{\left(m_{2} l_{2}^{2}+j_{2}\right)}+\frac{u m_{2} l_{1} l_{2} \cos x_{3}\left(\begin{array}{l}
m_{2}^{2} l_{1} l_{2}^{2} g \sin x_{3} \cos x_{3} \\
+m_{2} l_{2} l_{2} b_{2} x_{4} \cos x_{3} \\
+m_{2} l_{1} l_{2}\left(m_{2} l_{2}^{2}+j_{2}\right) \sin x_{3} \\
-b_{1}\left(m_{2} l_{2}^{2}+j_{2}\right) x_{2} \\
+\left(m_{2} l_{2}^{2}+j_{2}\right)
\end{array}\right)}{\left(\begin{array}{l}
m_{2}^{2} l_{1}^{2} l_{2}^{2}+m_{2} j_{2} l_{1}^{2} \\
+m_{2} l_{2}^{2} j_{1}+j_{1} j_{2}- \\
m_{2}^{2} l_{1}^{2} l_{2}^{2} \cos ^{2} x_{3}
\end{array}\right)\left(m_{2} l_{2}^{2}+j_{2}\right)}\right)
\end{aligned}
$$

The multitude of the single rotary inverted pendulum parameters are summarized in Table 1 [13]. It is convenient to present the linear standard form of the system [Eqs. (3) - (6)] in the following form.

$$
\dot{x}=A x+B u
$$

Table 1 Parameters for the single rotary inverted pendulum system.

\begin{tabular}{|c|c|}
\hline Parameter & Value \\
\hline$m_{2}$ & $0.075(\mathrm{~kg})$ \\
\hline$b_{1}$ & $0.0001(\mathrm{Nms})$ \\
\hline$b_{2}$ & $0.00028(\mathrm{Nms})$ \\
\hline$l_{1}$ & $0.148(\mathrm{~m})$ \\
\hline$l_{2}$ & $0.278(\mathrm{~m})$ \\
\hline$j_{1}$ & $2.48\left(10^{-2}\right)\left(\mathrm{kgm}^{2}\right)$ \\
\hline$j_{2}$ & $3.86\left(10^{-3}\right)\left(\mathrm{kgm}^{2}\right)$ \\
\hline$g$ & $9.81\left(\mathrm{~m} / \mathrm{s}^{2}\right)$ \\
\hline
\end{tabular}

The inverted pendulum system is linearized around the equilibrium point, $x=\left[\begin{array}{llll}0 & 0 & 0 & 0\end{array}\right]^{T}$, thus, the SSR can be obtained as:

$$
A=\left[\begin{array}{cccc}
0 & 1 & 0 & 0 \\
0 & -0.0035 & 2.222 & 0.0054 \\
0 & 0 & 0 & 1 \\
0 & 0.0019 & -21.034 & -0.0539
\end{array}\right], B=\left[\begin{array}{c}
0 \\
34.6425 \\
0 \\
-19.4264
\end{array}\right]
$$

At this point, it is important to validate the controllability of the proposed system at the equilibrium point. The controllability matrix $C_{0}$ of the SSR model can be obtained as follows [15],

$$
C_{0}=\left[\begin{array}{llll}
B & A B & A^{2} B & A^{3} B
\end{array}\right]
$$

Hence, the controllability matrix can be computed as shown in Eq. (10).

$$
C_{0}=\left[\begin{array}{cccc}
0 & 34.642 & -0.225 & -43.163 \\
34.642 & -0.225 & -43.163 & 4.849 \\
0 & -19.426 & 1.115 & 408.563 \\
-19.426 & 1.115 & 408.563 & -45.573
\end{array}\right]
$$

The linearized model is obviously controllable due to the existence of a full-row rank $(n=4)$ in the controllability matrix, $C_{0}$. After confirming that the single rotary inverted pendulum can be stabilized by the motor torque at the steady-state point, the analytical algorithm was utilized to formulate the pendulum angle and angular velocity profiles. Then, the link angle and angular velocity profiles are numerically generated by selecting one free variable while the motor torque is analytically determined based on the link angular velocity and time. As much as the proposed analytical function awards a conformable reference pendulum angle to the real profile, the reference torque confirms remarkably the stability of the system with no closed-loop command as it will be discussed in details in the next section.

\section{Analytical Algorithm}

Usually, the main objective of converting the governing differential equations to the state space representation model is to determine the characteristic roots which are used to understand the nature of the system response. Then, a feedback control is designed either in linear or nonlinear methods to perform a good stability, damping, tracking, response speed and disturbance rejection. However, this new algorithm does not rely on any linear or nonlinear control methods. Instead, an analytical control method is employed to stabilize the rotary inverted pendulum. In this work, the single rotary inverted pendulum utilizes a single pendulum angle profile. This reference profile is parameterized by a six-term exponential function of time which is given in Eq. (11).

$$
x_{3 \mathrm{ref}}=\sum_{n=1}^{6} c_{n} \exp (-n t)
$$


Taking the derivative of Eq. (11) with respect to time produces an expression for the reference pendulum velocity profile:

$$
\dot{x}_{3 \text { ref }}=\sum_{n=1}^{6}-n c_{n} \exp (-n t)=x_{4 \text { ref }}
$$

Similarly, an expression for the reference pendulum acceleration profile can be determined by taking the derivative of Eq. (12) with respect to time.

$$
\dot{x}_{4 \mathrm{ref}}=\sum_{n=1}^{6} n^{2} c_{n} \exp (-n t)
$$

Substituting Eqs. (11), (12), and (13) into Eq. (6) and solving the last equation for $u$, the reference motor torque is obtained which is given in Eq. (14).

$$
u_{\mathrm{ref}}=-\frac{\left(\begin{array}{l}
0.5 m_{2}^{2} l_{1}^{2} l_{2}^{2} \sin \left(2\left(\sum_{n=1}^{6} c_{n} \exp (-n t)\right)\right. \\
+\left(m_{2} g l_{2} j_{1}+g l_{1}^{2} l_{2} m_{2}^{2}\right) \sin \left(\sum_{n=1}^{6} c_{n} \exp (-n t)\right) \\
-m_{2} l_{1} l_{1} b_{1} x_{2 \mathrm{rec}} \cos \left(\sum_{n=1}^{6} c_{n} \exp (-n t)\right) \\
-m_{2}^{2} l_{1} l_{2}^{2}\left(\sum_{n=1}^{6} n^{2} c_{n} \exp (-n t)\right) \cos ^{2}\left(\sum_{n=1}^{6} c_{n} \exp (-n t)\right)+m_{2}^{2} g_{1}^{2} l_{2}+ \\
\left(m_{2} b_{2} l_{1}^{2}+b_{2} j_{1}\right)\left(\sum_{n=1}^{6}-n c_{n} \exp (-n t)\right) \\
+\left(m_{2}^{2} l_{1}^{2} l_{2}^{2}+m_{2} j_{1} l_{2}^{2}+m_{2} j_{2} l_{1}^{2}+j_{1} j_{2}\right)\left(\sum_{n=1}^{6} n^{2} c_{n} \exp (-n t)\right)
\end{array}\right.}{m_{2} l_{2} l_{2} \cos \left(\sum_{n=1}^{6} c_{n} \exp (-n t)\right)}
$$

From Eq. (14 ), it can be seen that the reference torque is a function of the reference link velocity $\left(x_{\text {2ref }}\right)$ and time $(t)$. Next, substituting Eq. (14) into Eq. (4), the reference link acceleration is obtained as shown in Eq. (15).

$$
\dot{x}_{2 \mathrm{eff}}=-\frac{\left(\begin{array}{l}
m_{2} g l_{2} \sin \left(\sum_{n=1}^{6} c_{n} \exp (-n t)\right)+b_{2}\left(\sum_{n=1}^{6}-n c_{n} \exp (-n t)\right) \\
+\left(m_{2} l_{2}^{2}+j_{2}\right)\left(\sum_{n=1}^{6} n^{2} c_{n} \exp (-n t)\right)
\end{array}\right)}{m_{2} l_{1} l_{2} \cos \left(\sum_{n=1}^{6} c_{n} \exp (-n t)\right)}
$$

Taking the first and second integrals of Eq. (15) with respect to time produces expressions for the reference link angular velocity and displacement profiles, respectively.

Six boundary conditions are needed to compute the six coefficients $c_{n}$. Here we used one pair of pendulum angle and velocity at the beginning time, one pair of pendulum angle and velocity and single pendulum acceleration at the steady-state time, and eventually, single of pendulum angle at three time positions and solve the system of six linear equations defined by Eqs. (11), (12) and (13). By definition, the single rotary pendulum begins to swing up from rest at $t(=0)$; the initial pendulum angle $\left(\theta_{2-0}\right)$ and initial pendulum velocity $\left(\dot{\theta}_{2-0}(=0)\right)$ are known parameters. The system reaches steady-state upright conditions at $t=1.5 \mathrm{sec}$ and its steady state pendulum angle $\theta_{2-s \mathrm{~s}}(=0)$, angular velocity $\dot{\theta}_{2-s \mathrm{~s}}(=0)$, and angular acceleration $\ddot{\theta}_{2-s \mathrm{~s}}(=0)$ are also known parameters. Finally, the additional boundary condition is required to compute the sixth exponential coefficient, a single pendulum angle value at a certain time is needed to satisfy all the six pendulum angle coefficients. The pendulum at the quarter-way point in the pendulum angle profile was used as the additional boundary condition. The time of the quarter-way point is simply $t=(0+1.5) / 4$. Our algorithm iterates on only one free parameter; hence, the quarter-way pendulum angle $\theta_{2 \text {-quarter }}$ is the free variable to be determined by the second integral of Eq. (15) while satisfies the desired link angle $\theta_{1-\mathrm{ss}}(=0)$. It is unlikely that the forward integration calculations in the link angle that ensures the actual link angle is positioned at steady-state upright point. Therefore, the pendulum angle profile must be reshaped by adjusting the quarter-way pendulum angle. For this to be done, we considered two values of initial pendulum angles $\left(\theta_{2-0}=20^{\circ}\right)$ and $\left(\theta_{2-0}=-20^{\circ}\right)$.

Figures 2 and 3 present reference state and input profiles vs. time for two initial pendulum angles; 20 deg and $-20 \mathrm{deg}$, respectively, and for different quarter-way pendulum angles; $\theta_{2 \text {-quater }}=50 \% \theta_{2-0}, \theta_{2 \text {-quarter }}=34 \% \theta_{2-0}$, $\theta_{2 \text {-quarer }}=21 \% \theta_{2-0}, \theta_{2-\text { quarer }}=16.7 \% \theta_{2-0}$, and $\theta_{2-\text { quarter }}=0 \% \theta_{2-0}$ deg. For both Figs. 2 and 3, Figures (a) - (f) show pendulum angle, pendulum angular velocity, pendulum angular acceleration, link angle, link angular velocity, and motor torque profiles. Figures 2a-2c and 3a-3c show that the single rotary inverted pendulum exhibits more swing up with selecting $(50 \%)$ of the initial pendulum angle, while the system with $(0 \%)$ of the initial pendulum angle performs with a less swing up. Figures 2d, 2e, 3d, and $3 \mathrm{e}$ show the link angle and link angular velocity computed by numerically integrating these system references forwards from the initial time to the steady-state time for two initial pendulum angles 20 deg and -20 deg, respectively. Clearly, Figs 2 and 3 show that selecting the quarter-way pendulum angle slightly less than $\left(34 \% \theta_{2-0}\right.$ deg) will shape the 
system references so that the link angle states are satisfied. For this scenario, $\theta_{2-\text { quarter }}=21 \% \theta_{2-0}$ generates system references so that the link angles at the initial and final time are achieved. The quarter-way pendulum angle is determined by a simple "brute-force" method: forward-link angle profiles are computed for a small number of trial values for the quarter-way pendulum angle and the steady-state link angle values are interpolated to compute the quarter pendulum angle that produces the desired link angle $\theta_{1}(=0) \mathrm{deg}$. Four trials were used here, and evaluating the required quarter pendulum angle is performed as long as the trial paths bracket the actual link angle $\theta_{1}(=0) \mathrm{deg}$.

To demonstrate this process, we introduced a one-dimensional cost function (J) which can be defined as the residual angle between the actual link angle $\left(\theta_{1}(=0) \mathrm{deg}\right)$ and the simulated link angle that results from the second integral of (Eq.15) at steadystate upright point. The one-dimensional interpolations for the two initial pendulum angles, 20 deg and -20 deg, are shown in Figs 4-a and 4b, respectively.
Bracketing the target link angle is achieved by using four quarter-way pendulum angles in the range $0 \% \theta_{2-0}<\theta_{2-\text { quarter }}<50 \% \theta_{2-0}$ deg as shown in the blue lines of Figs. 2 and 3. While, the red profiles in both Figs 2 and 3, present the suitable profiles that stabilize the single rotary inverted pendulum while satisfying all the boundary conditions.

In summary, Equation (14) is the required motor torque command to track the reference pendulum angle, pendulum angular velocity, link angle and link angular velocity profiles defined by Eqs. (11), (12) and (15), respectively. Equation (15) is numerically integrated using a variable-step, ode45 method forwards from the initial time $t(=0)$, to the steadystate time $t=1.5 \mathrm{sec}$ to select the correct quarter pendulum angle that performs the desired steadystate angle.

Equations (11), (12), (14), (15) determine the pendulum angle, pendulum angular velocity, motor torque, link angle, and the link angular velocity during the numerical simulation.

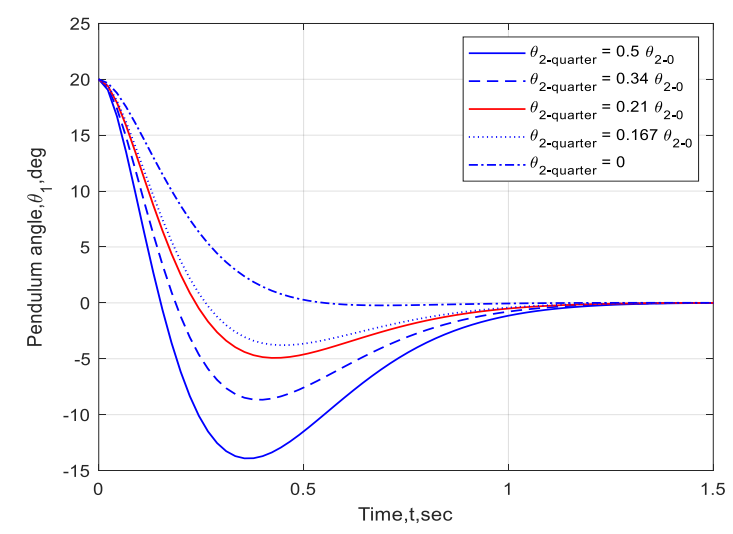

(a)

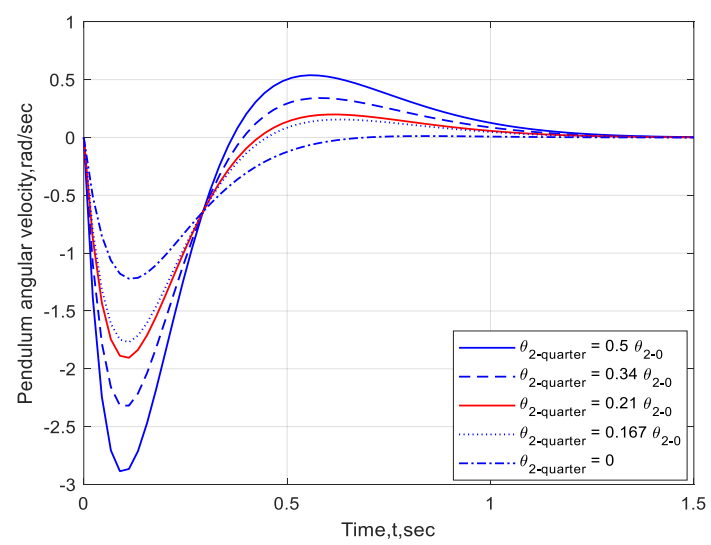

(b) 


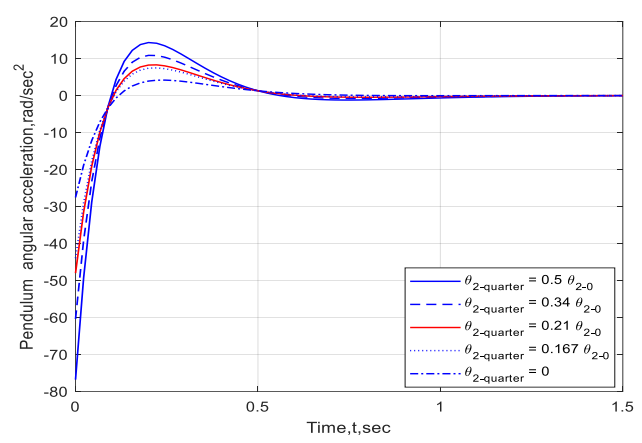

(c)

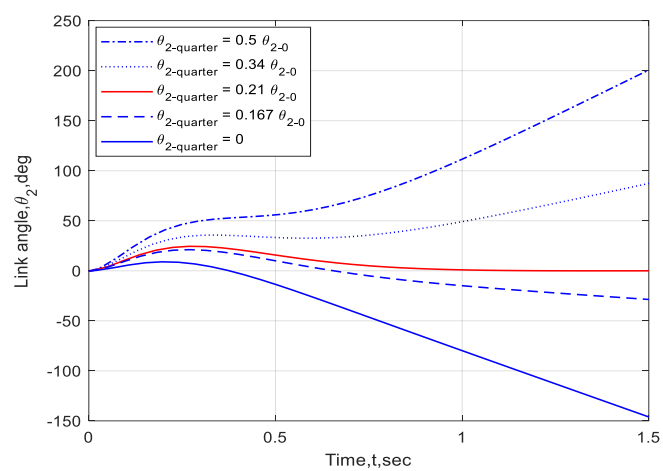

(d)

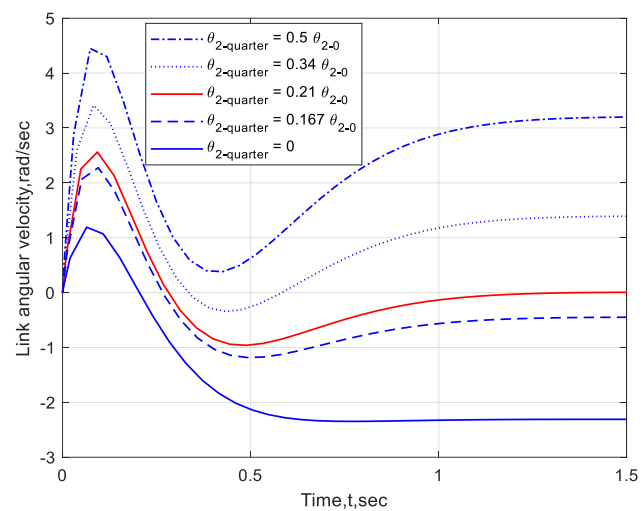

(e)

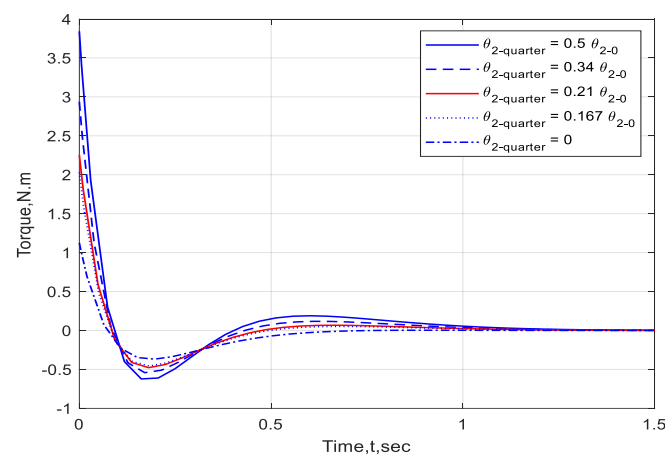

(f)

Figure 2: Reference state and input profiles for initial pendulum angle $\left(\theta_{2-0}=20^{\circ}\right)$ for different quarter-way pendulum angles; (a) pendulum angle vs. time; (b) pendulum angular velocity vs. time; (c) pendulum angular acceleration vs. time; (d) link angle vs. time; (e) link angular velocity vs. time; (f) motor torque vs. time. 


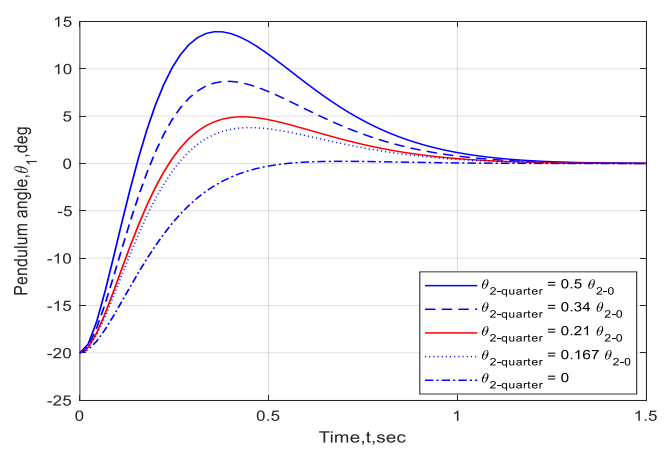

(a)

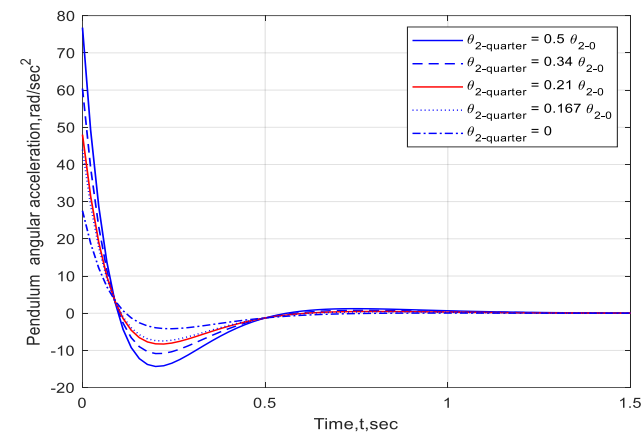

(c)

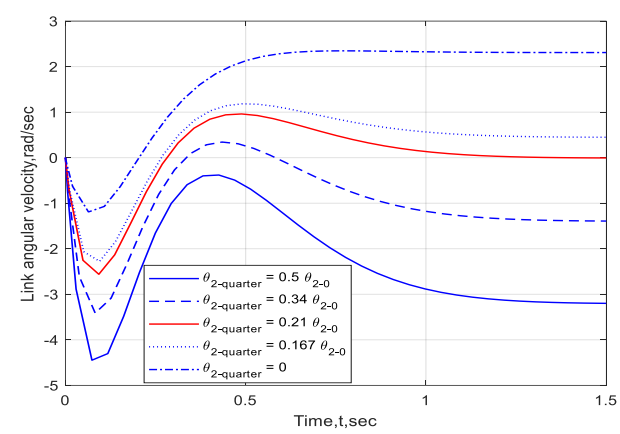

(e)

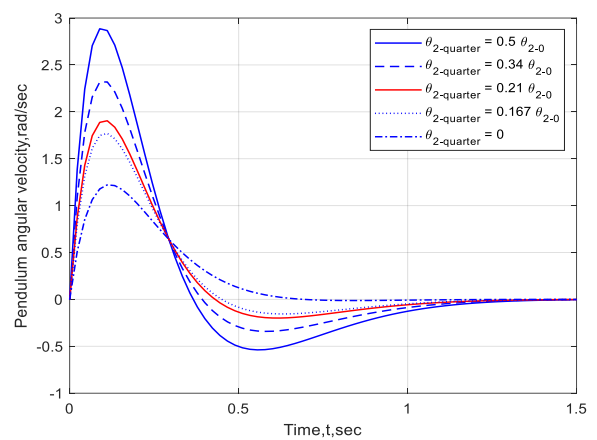

(b)

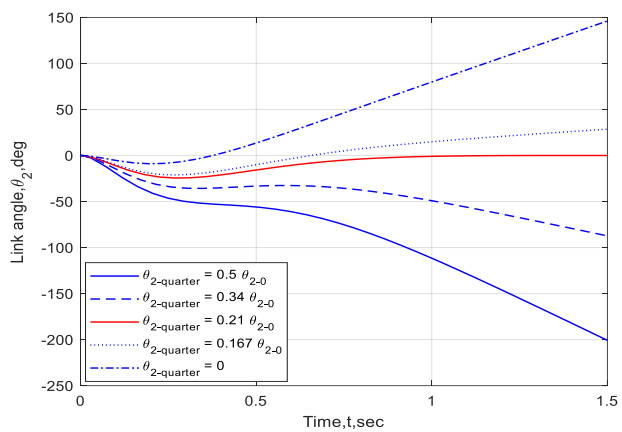

(d)

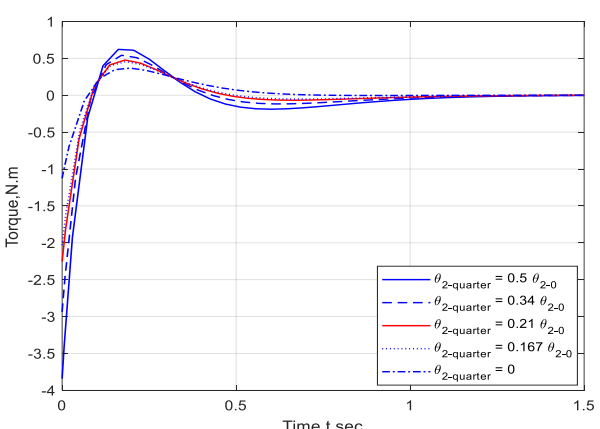

(f)

Figure 3: Reference state and input profiles for initial pendulum angle $\left(\theta_{2-0}=-20^{\circ}\right)$ for different quarter-way pendulum angles; (a) pendulum angle vs. time; (b) pendulum angular velocity vs. time; (c) pendulum angular acceleration vs. time; (d) link angle vs. time; (e) link angular velocity vs. time; (f) motor torque vs. time

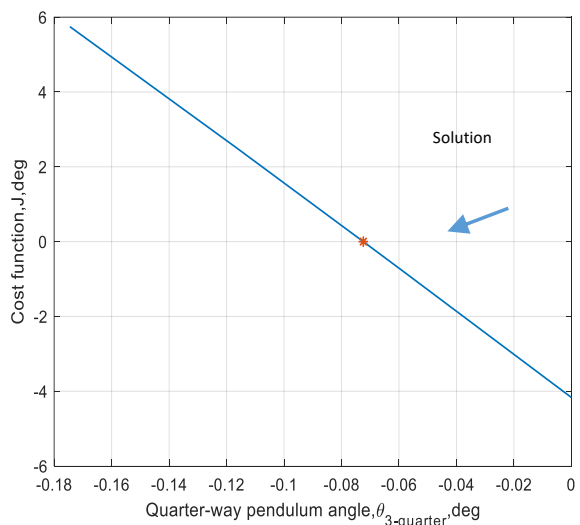

(a)

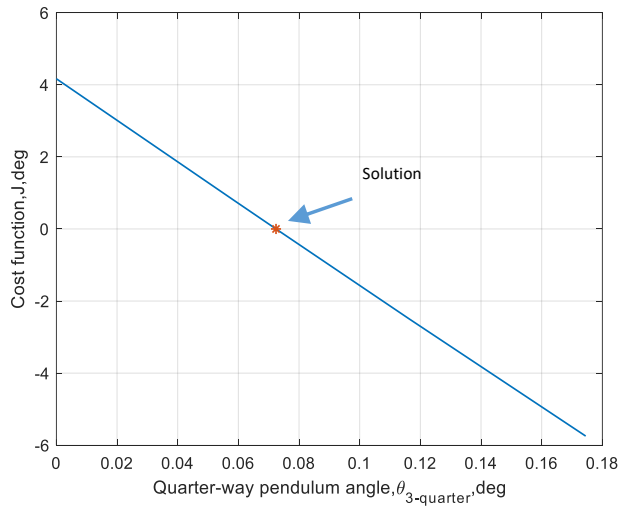

(b)

Figure 4:1-D search; (a) initial pendulum angle =20 deg; (b) initial pendulum angle= $-20 \mathrm{deg}$. 


\section{Actual Simulation}

The objective of pendulum algorithms is to stabilize and control the swing-up of the inverted pendulum. In general, the algorithm relies on two principles: shaping a reference trajectory, and then designing a closed-loop command to keep tracking the generated reference trajectory. However, in this work, generating a reference trajectory with no control effort is adequate to accurately stabilize the system.

As previously mentioned, the reference pendulum angle and reference pendulum angular velocity profiles were shaped analytically using Eqs. 12 and 13, respectively. While the reference link angle and reference link angular velocity profiles were generated numerically using Eq. 15.

The reference torque profile was computed analytically using Eq. 14. Because of the proposed exponential function provides a congruent reference

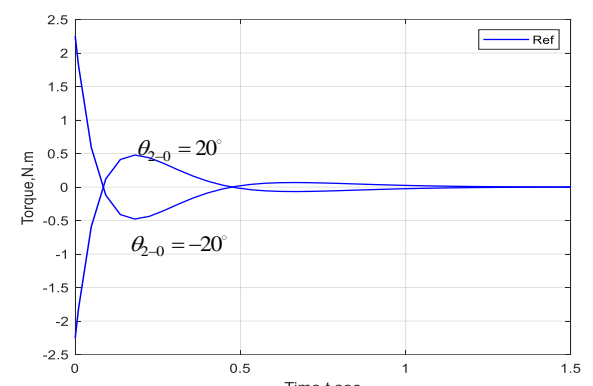

(a)

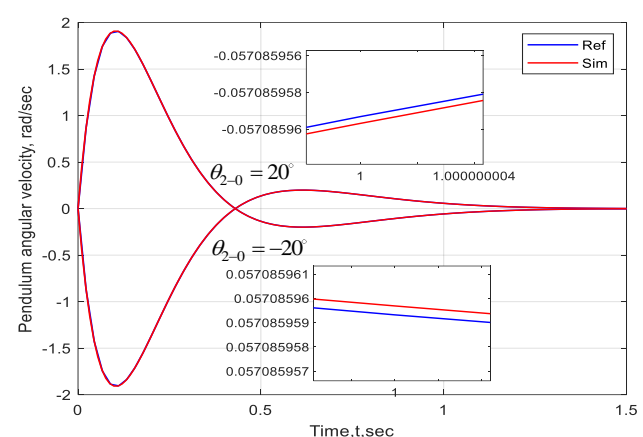

(c)

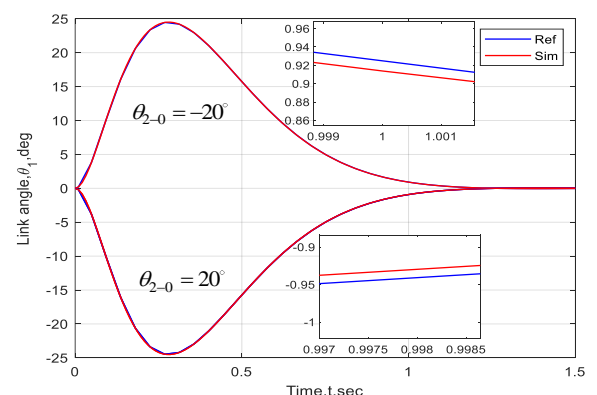

(e) pendulum angle with the real profile, the reference torque was used to control the single rotary inverted pendulum [Eqs. (3) - (6)] and guarantee stability of the system with no extra control effort.

Figures 5(a)-5(f), show the motor torque, pendulum angle, pendulum angular velocity, pendulum angular acceleration, link angle, and link angular velocity, respectively. These profiles were obtained for two initial pendulum angles, maximum value of $20 \mathrm{deg}$ and minimum value of $-20 \mathrm{deg}$. Figure 5(a) presents the reference torque profile which was determined analytically using Eq. 14 after adjusting the quarter pendulum angle.

The analytical controller, motor torque was then used to stabilize the single rotary inverted pendulum without utilizing any linear or nonlinear control methods. Figures 5(b)-5(f) show that the pendulum angle, pendulum angular velocity, pendulum angular acceleration, link angle, and link angular velocity references are tracked very precisely without any closed-loop command.

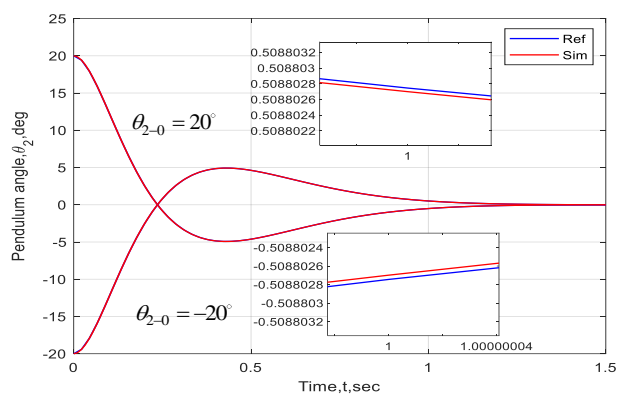

(b)

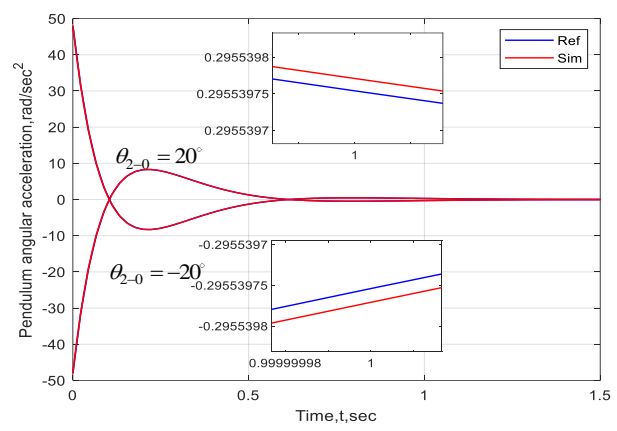

(d)

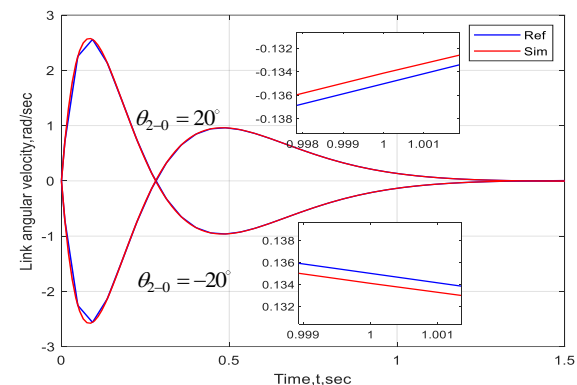

(f)

Figure 5: Reference and actual state and input profiles for two initial pendulum angle $\left(\theta_{2-0}=20^{\circ}\right)$ and $\theta_{2-0}=-20^{\circ} ;($ a) reference torque vs. time (b) pendulum angle vs. time; (c) pendulum angular velocity vs. time; (d) pendulum angular acceleration vs. time; (e) link angle vs. time; (f) link angular velocity vs. time. 
As shown in Fig 5, all the states reach the steadystate upright position with short time and small oscillations, which indicates the ability of the analytical algorithm to perform both swing-up and stabilization shortly. However, it is unlikely to adequately confirm the effectiveness of the proposed algorithm based on two initial pendulum angles.

For this reason, random initial pendulum angles restricted between 20 and -20 deg were addressed using Monte-Carlo simulation. The Monte-Carlo simulation is discussed in the next section which demonstrated that the rotary pendulum system can be stabilized while handling very large dispersions in the initial pendulum angle.

\section{Monte-Carlo Simulation Method}

The Monte-Carlo simulation is a method that relies on repeated random parameters to involve uncertainties in a system dynamics and to understand the behavior of the system under these critical conditions. Common statistics results such as the standard deviation, $S_{d}$, and mean value, $\bar{\mu}$, were used to analyze the algorithm results [16] and [17].

In this work, 1000 Monte-Carlo dispersed simulations were considered to evaluate the performance of the new proposed online analytical algorithm in the presence of wide dispersions in initial pendulum angle. The initial pendulum angles were adjusted between -20 and $20 \mathrm{deg}$.

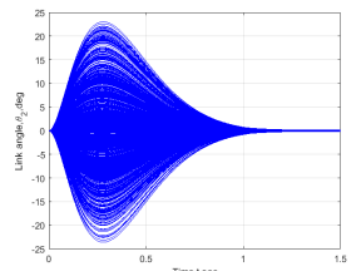

(a)

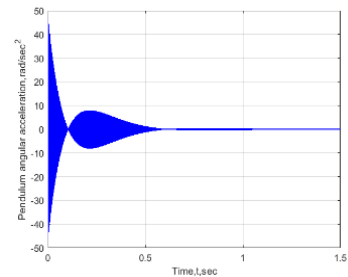

(c)

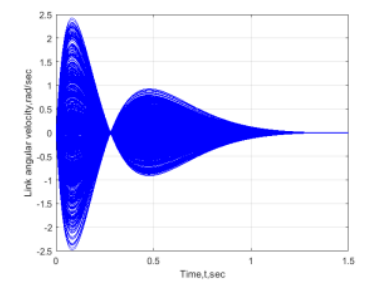

(e)
Figures 6(a)-6(f) present the 1000 pendulum angle, pendulum angular velocity, pendulum angular acceleration, link angle, link angular velocity, and motor torque histories, respectively. These figures show that the reference trajectories are accurately tracked by the actual ones with using the reference motor torque only.

Figure 6(f) indicates that the maximum and minimum required motor torque are bounded between 2.1 and -2.1 N.m, respectively, for all the simulated trajectories, hence the pendulum system can perfectly achieve the steady state upright position without excessive motor torque.

Figures 7(a)-7(e) show the statistical results of the pendulum angle, pendulum angular velocity, pendulum angular acceleration, link angle, link angular velocity errors, respectively, at the steadystate upright position. It can be noted from Fig. 7 that all steady-state errors are quite small. The statistics of all steady-state upright errors are summarized in Table 2.

Figure 7 and Table 2 show a summary of the Monte-Carlo simulations. These statistical results demonstrate that the minimum and maximum values match well to the desired conditions at the steadystate upright position, and all the standard deviation values are effectively zero. Hence, these results show the ability of the designed algorithm to perform both swing-up and stabilization shortly under wide range of initial pendulum dispersions.

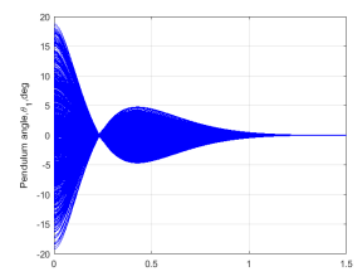

(b)

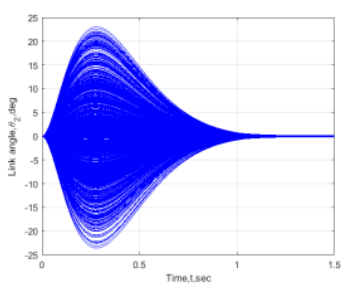

(d)

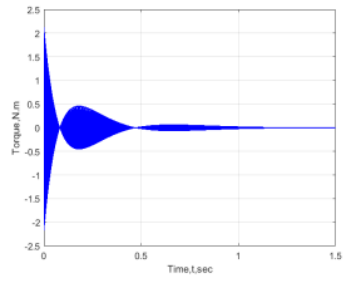

(f)

Figure 6: Single rotary inverted pendulum profiles for 1000 Monte-Carlo trials; (a) pendulum angle vs. time; (b) pendulum angular velocity vs. time; (c) pendulum angular acceleration vs. time; (d) link angle vs. time; (e) link angular velocity vs. time; (f) motor torque vs. time 

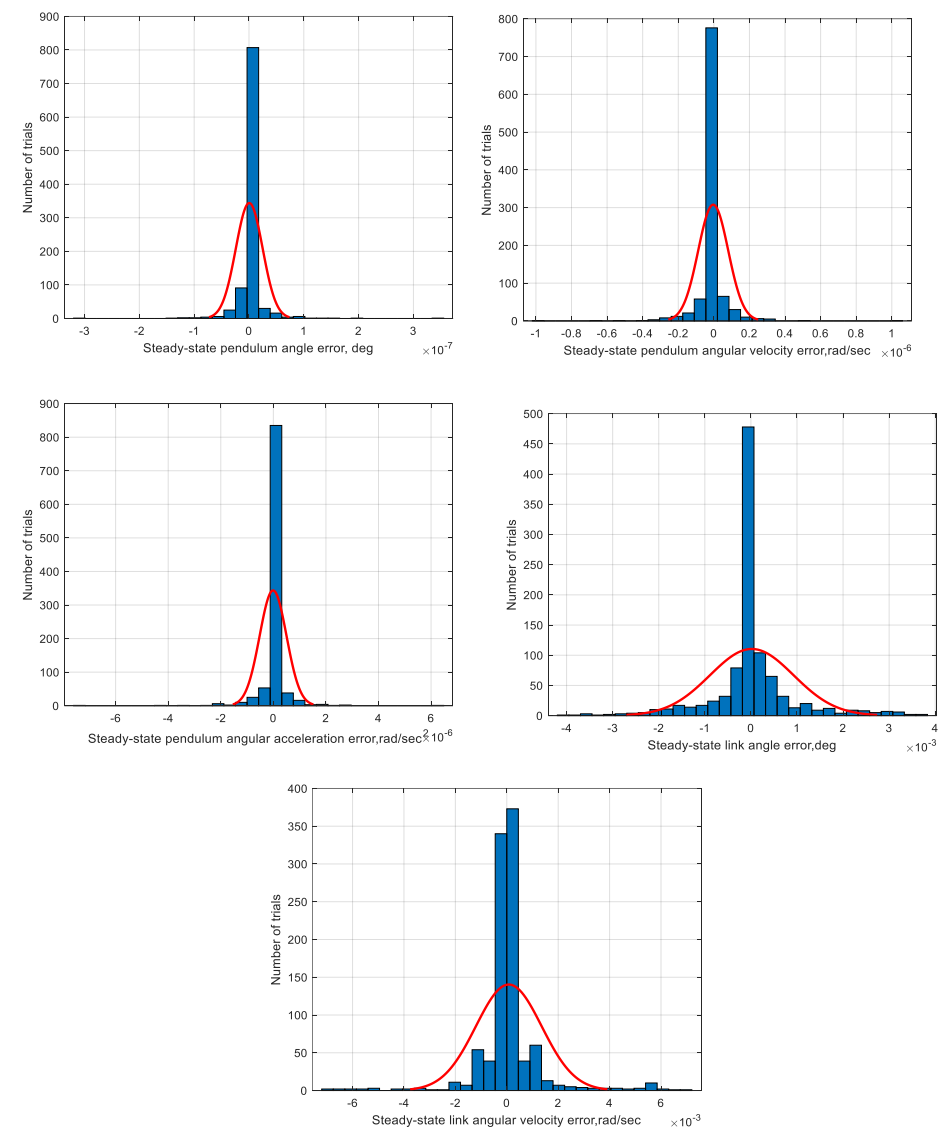

Figure 7. Histograms of steady-state errors from 1000 Monte-Carlo trials; (a) pendulum angle errors; (b) pendulum angular velocity errors; (c) pendulum angular acceleration errors; (d) link angle errors; (e) link angular velocity

Table 2 Statistics for steady-state upright state errors.

\begin{tabular}{|c|l|l|l|l|}
\hline State error & Mean & $\begin{array}{l}\text { Standard } \\
\text { deviation }\end{array}$ & \multicolumn{1}{|c|}{ Min } & Max \\
\hline $\begin{array}{c}\text { Pendulum } \\
\text { angle (deg) }\end{array}$ & $\begin{array}{l}4.8 \\
\left(10^{-10}\right)\end{array}$ & $\begin{array}{l}2 . \\
\left(10^{-8}\right)\end{array}$ & $\begin{array}{l}-3 . \\
\left(10^{-7}\right)\end{array}$ & $\begin{array}{l}3.5 \\
\left(10^{-7}\right)\end{array}$ \\
\hline $\begin{array}{c}\text { Pendulum } \\
\text { angular } \\
\text { velocity } \\
\text { (rad/sec) }\end{array}$ & $\begin{array}{l}-3.5 \\
\left(10^{-9}\right)\end{array}$ & $\begin{array}{l}8.4 \\
\left(10^{-8}\right)\end{array}$ & $\begin{array}{l}-9.8 \\
\left(10^{-7}\right)\end{array}$ & $\begin{array}{l}1 \\
\left(10^{-6}\right)\end{array}$ \\
\hline $\begin{array}{c}\text { Pendulum } \\
\text { angular } \\
\text { acceleration } \\
\text { (rad/sec })\end{array}$ & $\begin{array}{l}-1 \\
\left(10^{-5}\right)\end{array}$ & $\begin{array}{l}5.1 \\
\left(10^{-7}\right)\end{array}$ & $\begin{array}{l}-7.4 \\
\left(10^{-6}\right)\end{array}$ & $\begin{array}{l}6.3 \\
\left(10^{-6}\right)\end{array}$ \\
\hline $\begin{array}{c}\text { Link } \\
\text { angle (deg) }\end{array}$ & $\begin{array}{l}1.9 \\
\left(10^{-5}\right)\end{array}$ & $\begin{array}{l}9 . \\
\left(10^{-4}\right)\end{array}$ & -0.0041 & 0.038 \\
\hline $\begin{array}{c}\text { Link } \\
\text { angular } \\
\text { velocity } \\
\text { (rad/sec) }\end{array}$ & $\begin{array}{l}7.7 \\
\left(10^{-5}\right)\end{array}$ & 0.0013 & -0.072 & 0.007 \\
\hline
\end{tabular}

\section{Conclusion}

An online stabilization method has been developed for a single rotary inverted pendulum system. The new analytical algorithm employs a single reference pendulum angle that is parameterized by a six-term exponential function. The tracking reference profiles were obtained by selecting a single free variable which is the pendulum angle at the quarter-way time while achieving all the boundary conditions.
Since the proposed exponential function provides a congruent reference pendulum angle profile with the actual profile, the reference motor torque has performed to be reliable and convenient to stabilize the system with no excessive effort. Monte-Carlo simulation results demonstrated the effectiveness of the proposed algorithm to perform both the swingup and the stabilization shortly despite the presence of a wide range of dispersions in the initial pendulum angle.

\section{References}

[1] Jadlovský, S. and Sarnovský, J., 2013. Modelling of classical and rotary inverted pendulum systemsa generalized approach. Journal of Electrical Engineering, 64(1), pp.12-19.

[2] Jadlovska, S. and Sarnovsky, J., 2013. A complex overview of modeling and control of the rotary single inverted pendulum system. Advances in Electrical and Electronic Engineering, 11(2), pp.73-85.

[3] Akhtaruzzaman, M. and Shafie, A.A., 2010, August. Modeling and control of a rotary inverted pendulum using various methods, comparative assessment and result analysis. In 2010 IEEE International Conference on Mechatronics and Automation (pp. 1342-1347). IEEE. 
[4] Wen, J., Shi, Y. and Lu, X., 2017. Stabilizing a rotary inverted pendulum based on logarithmic Lyapunov function. Journal of Control Science and Engineering, 2017.

[5] Wen, J., Shi, Y. and Lu, X., 2017, May. Stabilizing a rotary inverted pendulum based on Lyapunov stability theorem. In 2017 29th Chinese Control And Decision Conference (CCDC) (pp. 56525657). IEEE.

[6] Mathew, N.J., Rao, K.K. and Sivakumaran, N., 2013. Swing up and stabilization control of a rotary inverted pendulum. IFAC Proceedings Volumes, 46(32), pp.654-659.

[7] Öksüz, M., Önal, M.B., Halicioğlu, R. and Dülger, L.C., 2018. Alternative controller design for rotary inverted pendulum. Tehnički glasnik, 12(3), pp.139-145.

[8] Kumar, K.P. and Rao, G.V.S.K., 2014, October. Modeling and controller designing of rotary inverted pendulum (RIP)-comparison by using various design methods. In Proceedings of International Journal of Science, Engineering and Technology Research (IJSETR) (Vol. 3, No. 10).

[9] Diao, X., Modular Control of a Rotary Inverted Pendulum System, (2016).

[10] Chawla, I. and Singla, A., 2018. Real-time control of a rotary inverted pendulum using robust LQRbased ANFIS controller. International Journal of Nonlinear Sciences and Numerical Simulation, 19(3-4), pp.379-389.
[11] Khanesar, M.A., Teshnehlab, M. and Shoorehdeli, M.A., 2007, June. Sliding mode control of rotary inverted pendulm. In 2007 Mediterranean Conference on Control \& Automation (pp. 1-6). IEEE.

[12] Rigatos, G., Siano, P., Abbaszadeh, M. and Ademi, S., 2017, July. Nonlinear H-infinity control for the rotary pendulum. In 2017 11th International Workshop on Robot Motion and Control (RoMoCo) (pp. 217-222). IEEE.

[13] Driver, J. and Thorpe, D., 2004. Design, build and control of a single/double rotational inverted pendulum. The University of Adelaide, School of Mechanical Engineering, Australia, 4.

[14] Hassanzadeh, I. and Mobayen, S., 2011. Controller design for rotary inverted pendulum system using evolutionary algorithms. Mathematical Problems in Engineering, 2011.

[15] Li, B., 2013. Rotational Double Inverted Pendulum (Doctoral dissertation, University of Dayton).

[16] Fawaz, A.B., Laheeb, M. and Salwan, K., 2018, January. Online algorithm for controlling an inverted pendulum system under uncertainty in design parameters and initial conditions using Monte-Carlo simulation. In 2018 IEEE 8th Annual Computing and Communication Workshop and Conference (CCWC) (pp. 1-7). IEEE.

[17] Laheeb, M., Salwan, K., Fawaz, A.B. and Sarah, L., 2018, January. Online algorithm for controlling a cruise system under uncertainty in design parameters and environmental conditions using Monte-Carlo simulation. In 2018 IEEE 8th Annual Computing and Communication Workshop and Conference (CCWC) (pp. 424-430). IEEE. 